\title{
Análise numérica da rigidez global do conjunto telha-terça em painéis laterais de aço
}

\section{Numeric analysis of the global stiffness of group purlin-sheeting in lateral steel panels}

\author{
Julio Cesar Molina ${ }^{1}$; Renato Bertolino Junior ${ }^{2}$
}

\section{Resumo}

Neste trabalho verificou-se numericamente o comportamento de painéis laterais de edifícios em aço, utilizando-se do conjunto telha-terça na contenção longitudinal. Considerou-se telhas trapezoidais, fixadas na $2^{\mathrm{a}}$ e $5^{\mathrm{a}}$ ondas baixas e telhas com todas as ondas baixas fixas. As telhas foram fixadas nas terças e este conjunto fixado aos pórticos do edifício, de maneira a garantir a sua funcionalidade como um painel rígido, ação diafragma. Foi também verificado o comportamento de painéis laterais, com sistema de contraventamento tradicional, efetuado por tirantes, sem a utilização do fechamento, tendo estes as mesmas dimensões dos painéis diafragma. Foram analisados painéis com dimensões 4,0, 5,0, 6,0 e 7,0 metros de comprimento, a partir da utilização do software SAP2000NonLinear. Como resultado, constatou-se que o aumento da rigidez proporcionada pela ação diafragma do conjunto telha-terça foi de até $84 \%$ com relação ao sistema contraventado tradicionalmente somente por tirantes.

Palavras-chave: Estruturas de aço. Contraventamento. Ação diafragma. Modelagem numérica.

\begin{abstract}
This paper presents an evaluation of the behaviour of lateral panels of steel buildings starting from computational modeling using the purlin-sheeting group in the longitudinal contention. The sheeting was fastened on $2^{\text {nd }}$ and $5^{\text {th }}$ waves and also in all waves. The sheeting was fastened in purlin and this system connected in the frame of the building to guarantee the functionality as a rigid panel, action diaphragm. It was also verified the behaviour of lateral panels, with traditional braced system using the same dimensions of the panels diaphragm. The panel was analyzed with dimensions of 4.0, 5.0, 6.0 and 7.0 meters, using the software SAP2000NonLinear. The results showed an increment of $84 \%$ related to the system traditional braced.
\end{abstract}

Key words: Steel structures. Braced. Action diaphragm. Computational modeling.

Professor da UNESP/ITAPEVA e Pós-doutorando da EESC/USP; juliocesarmolina@yahoo.com.br

2 Professor Titular da FEIS/UNESP; rbj@dec.feis.unesp.br 


\section{Introdução}

As construções metálicas de médio porte, tais como as edificações em aço, apresentam um desempenho estrutural eficiente. São basicamente compostas por elementos principais: pilares, vigas ou treliças, e por componentes secundários: terças, longarinas, contraventamentos e fechamentos. Esses elementos podem ser observados na Figura 1. Além disso, as edificações em aço têm como herança de concepção estrutural algumas características marcantes, tais como a utilização de contraventamentos para garantir a estabilidade global da estrutura durante sua vida útil, como também na fase de montagem, e de correntes para contenção lateral das terças, conforme ilustrado na Figura 2. Os contraventamentos tradicionalmente utilizados, juntamente com as terças e longarinas formam conjuntos treliçados nos planos vertical e do telhado, transferindo as ações horizontais para a fundação. Garante-se assim a estabilidade transversal e principalmente longitudinal da edificação.

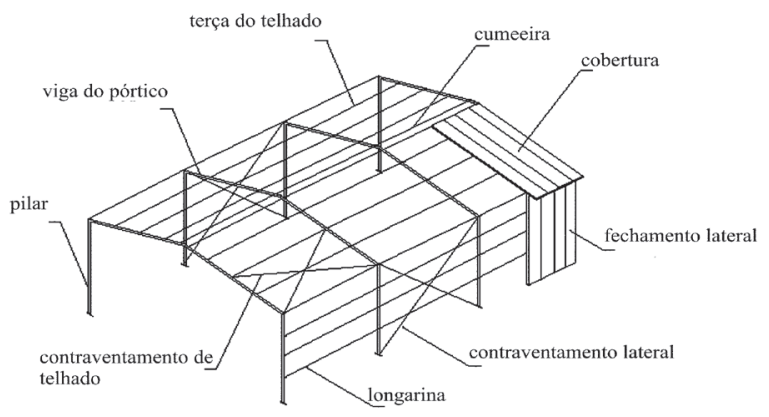

Figura 1. Componentes básicos de uma edificação em aço. Fonte: Adaptado de Bellei (1994).

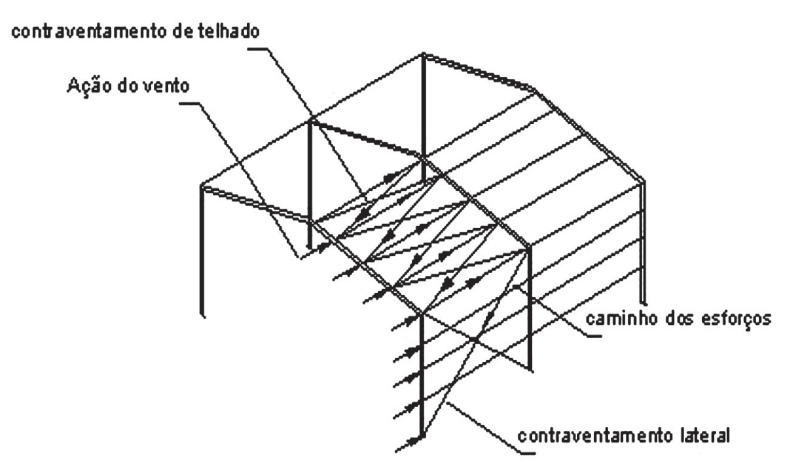

Figura 2. Contraventamento tradicional, efetuado por tirantes. Fonte: Adaptado de Bellei (1994).

No sistema de contraventamento tradicional, efetuado por tirantes, o deslocamento do painel lateral do edifício, provocado pela ação de uma força horizontal (por exemplo, ação do vento), gera esforços de tração na diagonal e de compressão no pilar, conforme indicado na Figura 3.

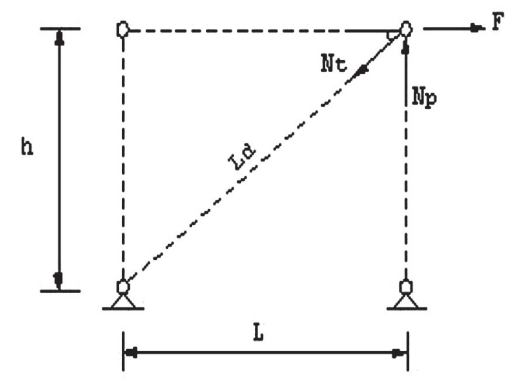

Figura 3. Esforços gerados em função da força horizontal F. Fonte: Adaptado de Englekirk (1994).

Assim, em função da ocorrência conjunta dos esforços $\mathrm{N}_{\mathrm{t}}$ e $\mathrm{N}_{\mathrm{p}}$, na diagonal e no pilar, respectivamente, ocorre um aumento $\left(\delta_{d}\right)$ da diagonal associado a um encurtamento $\left(\delta_{\mathrm{p}}\right)$ do pilar, conforme ilustrado na Figura 4. 


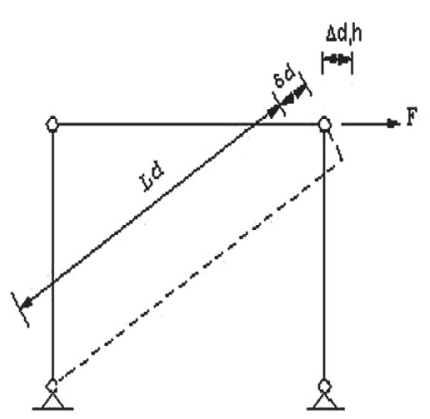

(a)

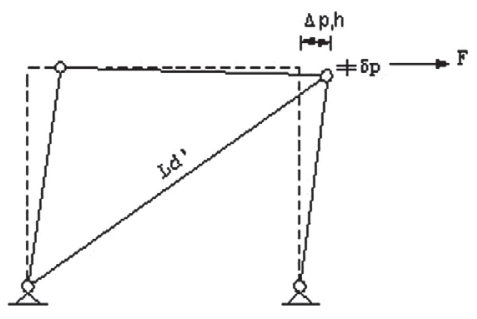

(b)

Figura4.Deslocamentoshorizontais:(a)Proporcionadopelo aumento da diagonal; (b) Proporcionado pelo encurtamento do pilar. Fonte: Adaptado de Englekirk (1994).

Os deslocamentos horizontais em função do aumento da diagonal de contraventamento e do encurtamento do pilar são expressos pelas equações (1) e (2), onde " $A_{d}$ " e "A " são respectivamente as áreas da diagonal do contraventamento e do pilar, sendo "E" o módulo de elasticidade do aço.

$$
\begin{aligned}
& \Delta_{d h}=F \cdot \frac{L_{d}{ }^{3}}{L^{2} \cdot A_{d} \cdot E} \\
& \Delta_{p h}=F \cdot \frac{h^{3}}{L^{2} \cdot A_{p} \cdot E}
\end{aligned}
$$

O deslocamento horizontal total $(\Delta)$ do sistema contraventado por tirantes para a força $F$ é dado por:

$$
\Delta=\Delta_{d h}+\Delta_{p h}=F \cdot\left(\frac{L_{d}{ }^{3}}{A_{d} \cdot E \cdot L^{2}}+\frac{h^{3}}{A_{p} \cdot E \cdot L^{2}}\right)
$$

A rigidez $(\mathrm{K})$ do sistema, neste caso, pode ser definida como:

$$
K=\frac{F}{\Delta}=\frac{A_{p} \cdot A_{d} \cdot E \cdot L^{2}}{A_{p} \cdot L_{d}{ }^{3}+A_{d} \cdot h^{3}}
$$

Além disso, nos projetos de estruturas, considerase que as ações laterais que atuam sobre um pórtico individual de edifício são resistidas por ele próprio. $\mathrm{Na}$ realidade uma parcela dessas ações pode ser transferida para os pórticos localizados nas extremidades pela cobertura através de esforços de cisalhamento. A esse mecanismo dá-se o nome de ação diafragma de cobertura. Segundo Baraldi (2001), na concepção estrutural, um diafragma pode ser considerado como uma viga especial, onde se tem, no caso do diafragma de cobertura, o momento resistido por um binário de forças de tração e compressão e o cisalhamento absorvido pelo diafragma, Figura 5.

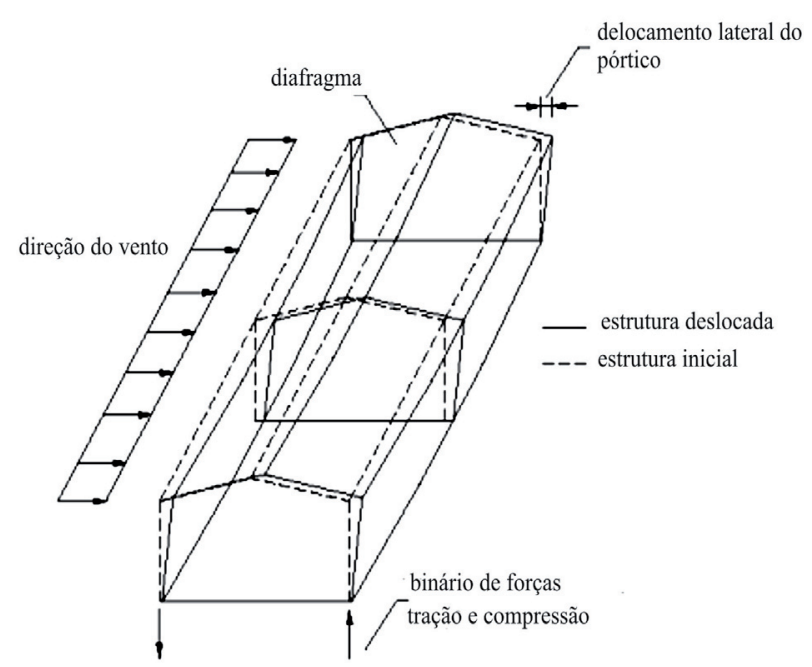

Figura 5. Comportamento da viga diafragma. Fonte: Adaptado de Baraldi (2001). 
No entanto, a ação diafragma pode ser considerada de cobertura ou de parede e é considerada para resistir no seu plano como um sistema de contraventamento, diminuindo os deslocamentos laterais. O diafragma de cobertura é formado principalmente pelas telhas e terças e o diafragma lateral pelas longarinas e contraventamentos. Devido ao efeito diafragma obtémse rigidez no plano resistente a ação horizontal que atua sobre a estrutura. Na prática, quando uma ação horizontal solicita a edificação, paralelamente ao seu comprimento, a ação diafragma ocorre no fechamento lateral. Assim, nas paredes lateriais de uma edificação em aço, observa-se que a ação diafragma atua como parte de um sistema de contraventamento, diminuindo o deslocamento horizontal $(\Delta)$, em função da rigidez proporcionada pela conexão telha-terça. A rigidez ao cisalhamento, $\left(G^{\prime}\right)$, para um painel diafragma pode ser obtida experimentalmente com base no modelo de $\mathrm{Yu}$ (1985), conforme Figura 6. Neste modelo, o conjunto telha-terça é submetido a uma força concentrada (F) que provoca o deslocamento $(\Delta)$ do painel na direção da força.

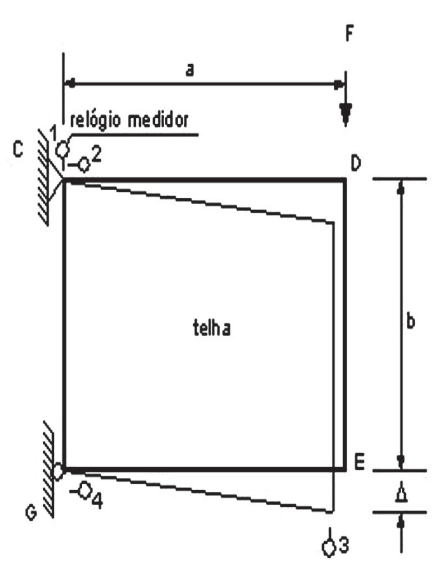

Figura 6. Cisalhamento da telha. Fonte: Adaptado de Yu (1985).

Os deslocamentos $\Delta_{1}, \Delta_{2}, \Delta_{3}$ e $\Delta_{4}$, indicados na Figura 6, segundo os pontos 1, 2, 3 e 4, são combinados para remover os efeitos da estrutura de suporte, onde "a/b" é a relação entre as dimensões do painel analisado.

$$
\Delta=\Delta_{3}-\left[\Delta_{1}+\frac{a}{b} \cdot\left(\Delta_{2}+\Delta_{4}\right)\right]
$$

A rigidez diafragma $\left(\mathrm{G}^{\prime}\right)$ é determinada com base na carga de aproximadamente $40 \%$ com relação a força última aplicada ao painel $\left(\mathrm{F}=0,4 \cdot \mathrm{F}_{\text {ult }}\right)$. O deslocamento lateral $(\Delta)$ é obtido, neste caso, pela combinação entre o deslocamento provocado por flexão $\left(\Delta_{\mathrm{f}}\right)$ e por cisalhamento $\left(\Delta_{\mathrm{s}}\right)$.

$$
\Delta_{\mathrm{s}}=\Delta-\Delta_{\mathrm{f}}
$$

O deslocamento devido a flexão é dado pela equação (7), onde I é o momento de inércia da armação do painel.

$$
\Delta_{f}=\frac{F \cdot a^{3}}{3 \cdot E \cdot I}
$$

$$
I=\frac{A \cdot b^{2}}{2}
$$

Portanto, a rigidez ao cisalhamento, (G'), considerando o fechamento composto pela telha é dada por:

$$
G^{\prime}=\frac{0,4 F_{u l t}}{\Delta_{s}} \cdot \frac{a}{b}
$$

O objetivo deste trabalho consiste na obtenção do acréscimo de rigidez proporcionada pela ação diafragma de parede, conjunto telha-terça, com relação a rigidez do sistema de contraventamento tradicional, efetuado por tirantes, considerando o espaçamento entre pórticos (L). 


\section{Materiais e métodos}

Foram analisados numericamente, a partir da utilização do software SAP2000NonLinear, painéis laterais de edifícios de aço. Os paineis foram analisados com e sem o fechamento lateral. Foram analisados painéis com dimensões longitudinais " $\mathrm{L}$ " de 4,00, 5,00, 6,00 e 7,00 metros, com altura "h" fixa de 4,90m. Para os painéis com fechamento foram analisados dois tipos de fixação para as telhas: fixação na $2^{\mathrm{a}}$ e $5^{\mathrm{a}}$ ondas baixas (nomeada por $2^{\mathrm{a}}$ e $5^{\mathrm{a}}$ ) e fixação em todas as ondas baixas (nomeada por TOF). Na composição dos pilares e vigas dos painéis foram utilizadas seções "I" de perfis de aço com dimensões 200x160x4,75mm. Nos contraventamentos diagonais foram utilizadas barras de aço circular com diâmetro de $12,7 \mathrm{~mm}$ e nas terças laterais (longarinas) perfis de aço com seções "U" de dimensões 100x50x2,65mm, espaçadas de $1,50 \mathrm{~m}$. Nos painéis com fechamento foram utilizadas telhas de aço, de seção trapezoidal, tipo MF40, com espessura $0,43 \mathrm{~mm}$, fixadas nas terças. As dimensões dos perfis de aço foram tomadas a partir do catálogo técnico da METFORM (2002a) e as dimensões das telhas a partir do catálogo da METFORM (2002b). Na modelagem dos painéis foram utilizados elementos de barra para representar as vigas, terças, pilares e contraventamentos, e elementos de casca para representaras telhas. O elemento de barra utilizado foi o elemento finito denominado "Frame element" sendo as seções transversais destes elementos disponibilizadas pela biblioteca interna do software. Este elemento possui dois nós, com três graus de liberdade por nó (referentes as translações) e responde a esforços axiais de tração e compressão. Os pilares foram discretizados em cinco divisões verticais e as terças discretizadas de modo que seus nós coincidissem com os nós das ondas baixas das telhas (a cada $196 \mathrm{~mm}$ ). Para os nós de fixação comuns entre telha e terça nenhum giro da telha foi considerado com relação a terça, tendo-se assim um conjunto rigido. As terças e vigas tiveram suas extremidades rotuladas nos contatos com os pilares. $\mathrm{Na}$ modelagem das telhas foi utilizado o elemento finito denominado "Shell element" que possui quatro nós e seis graus de liberdade por nó (três translações e três rotações), sendo as telhas discretizadas em três divisões verticais a cada duas terças. As Figuras 7 e 8 mostram as configurações dos elementos, como também as discretizações utilizadas.

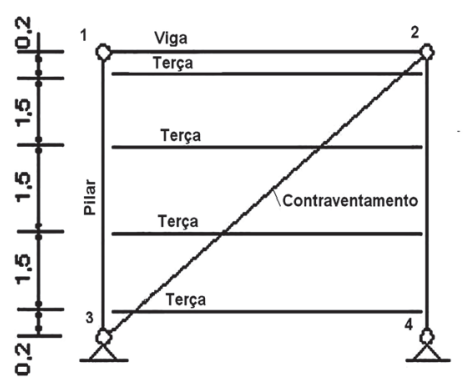

(a)

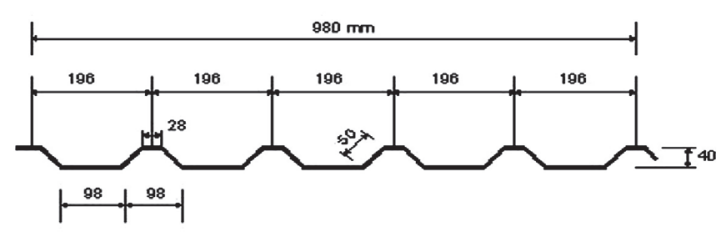

(b)

Figura 7. Configurações: (a) Painel lateral; (b) Telha.

Além disso, a costura entre duas telhas foi considerada continua e os pesos próprios dos elementos desconsiderados na análise dos painéis. As Figuras $8 \mathrm{a}$ e $8 \mathrm{~b}$ mostram, respectivamente, as configurações dos paineis analisados sem e com o fechamento de telha. Os painéis foram submetidos a carregamentos horizontais (F) de até $100 \mathrm{kN}$, para obtenção dos deslocamentos $(\Delta)$, e também para a verificação dos seus comportamentos, para os dois sistemas de contenção longitudinal analisados.

Os detalhes tridimensionais dos perfis que compõesos pilares, vigas, terçasecontraventamentos, e também das telhas que compõem o fechamento lateral dos painíes, estão apresentados nas Figuras $9 \mathrm{a}$ e $9 \mathrm{~b}$, respectivamente. 


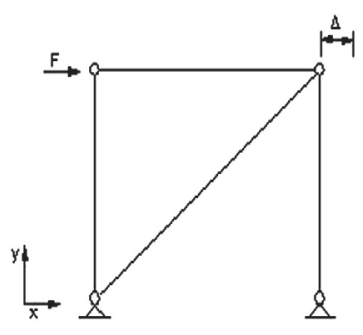

(a)

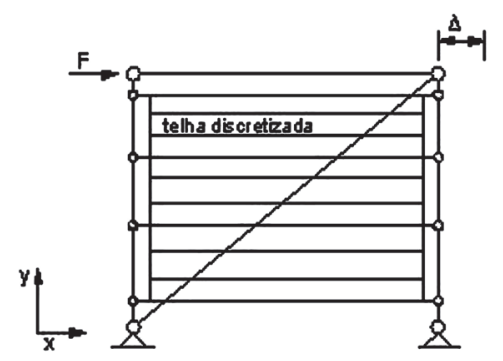

(b)

Figura 8. Painéis: (a) Sem fechamento lateral (nomeado por PSFL); (b) Com fechamentos laterais $\left(2^{\mathrm{a}}\right.$ e $\left.5^{\mathrm{a}}\right)$ e (TOF).

Com o objetivo de se verificar o acréscimo de rigidez proporcionado pela fixação telha-terça, ao sistema de contraventamento convencional de tirantes, foram determinados, inicialmente, valores de rigidez "K" e "G" para os painéis analisados, respectivamente, sem e com o fechamento lateral. Estes valores de rigidez foram obtidos a partir da relação direta entre a força horizontal (F) aplicada e o correspondente deslocamento $(\Delta)$ obtido. As simulações numéricas para obtenção dos deslocamentos $(\Delta)$, neste caso, foram efetuadas a partir da consideração de uma análise linear e também não linear geométrica dos painéis. O SAP2000NonLinear não considera a não linearidade fisica dos materiais. Vale mencionar ainda

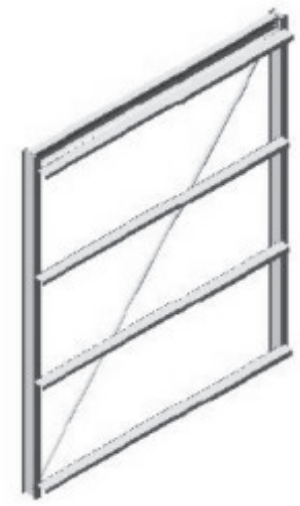

(a)

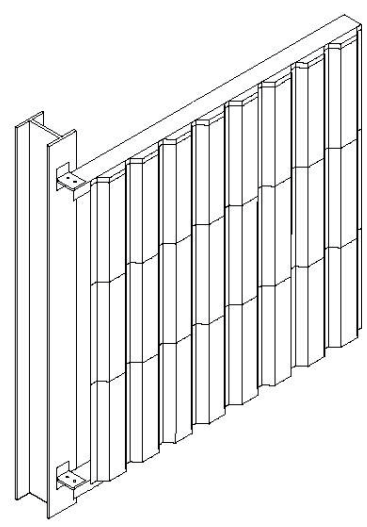

(b)

Figura 9. Elementos que compõem os painéis: (a) Pilares, viga, terças e contraventamento; (b) Posicionamento das telhas.

que o SAP2000NonLinear é um software mais voltado para fins comerciais e não possui os mesmos recursos de outros softwares também utilizados em análises numércias de estruturas como, por exemplo, o ANSYS e o ABAQUS, que embora possibilitem análises mais precisas de eventuais efeitos localizados, por outro lado exigem um esforço computacional mais elevado. Nestes softwares, é possivel a consideração da não linearidade física dos materiais a partir da definição de relações constitutivas para os mesmos. Basaglia (2004), apresenta um modelo numérico, construído no ANSYS, para verificação de aspectos localizados de interesse, no qual a relação constitutiva utilizada para o aço de perfis formados a frio, unidos a telhas 
de aço por meio de parafusos, é baseada num modelo elasto-plástico multilinear com encruamento isótropo, associado ao critério de plastificação de von Mises. No entanto, a análise numérica apresentada neste artigo, a partir da utilização do SAP2000NonLinear, é válida, pois, neste caso, estão sendo verificados apenas os aspectos relativos ao comportamento global dos paineis de aço, focalizando a rigidez proporcionada pelo conjunto telha-terça em adição a rigidez do sistema de contraventamento efetuado por tirantes. Além disso, com o objetivo de se avaliar a influência das dimensões " $L$ " dos painéis, conforme apresentadas na Figura 3, na rigidez global da estrutura, foram também determinados os valores de G', a partir da equação (9), tendo como base os deslocamentos $(\Delta)$, obtidos no SAP2000NonLinear para um carregamento horizontal definido em função do deslocamento $\left(\Delta_{\lim }\right)$, recomendado pela Associação Brasileira de Normas Técnicas (2001). Admitiu-se, neste caso, $\Delta_{\lim }=$ 12,05mm, $\left(\Delta_{\lim }=\mathrm{h} / 400\right.$, definido segundo (ABNT, 2001). Para esse valor limite foram obtidos os valores de força limite $\left(\mathrm{F}_{\text {lim }}\right)$ para cada configuração de painel analisada. Para a determinação do valor de G', os valores dos deslocamentos provocados por cisalhamento $\left(\Delta_{\mathrm{s}}\right)$ e por flexão $\left(\Delta_{\mathrm{f}}\right)$ foram obtidos a partir da utilização das equações (6) e (7), respectivamente, sendo os momentos de inércia das armações dos painéis obtidos pela equação (8), com base na área média das seções dos perfis de aço, correspondentes as barras CD e GE, conforme indicadas na Figura 6.

\section{Resultados}

Os resultados das análises numéricas linear (nomeada por AL) e não linear geométrica (nomeada por ANL) dos painéis, realizadas no SAP2000NonLinear, são apresentados nas tabelas 1 a 10 e nas figuras 10 a 14 .
Tabela 1. Painéis sem fechamento lateral (PSFL) - AL.

\begin{tabular}{lllll}
\hline $\mathrm{F}$ & $\Delta \mathbf{( m m})$ & & & \\
\cline { 2 - 5 }$(\mathrm{kN})$ & $\mathbf{L}=\mathbf{4 m}$ & $\mathbf{L}=\mathbf{5 m}$ & $\mathbf{L}=\mathbf{6 m}$ & $\mathbf{L}=\mathbf{7 m}$ \\
\hline 100 & 62,6 & 53,8 & 49,9 & 46,5 \\
\hline
\end{tabular}

Tabela 2. Painéis com fechamento lateral - AL.

\begin{tabular}{|c|c|c|c|c|}
\hline \multirow{3}{*}{$\begin{array}{l}\mathrm{F} \\
(\mathrm{kN})\end{array}$} & \multicolumn{4}{|l|}{$\Delta(\mathrm{mm})$} \\
\hline & \multicolumn{2}{|c|}{$L=4 m$} & \multicolumn{2}{|l|}{$\mathbf{L}=\mathbf{5 m}$} \\
\hline & $2^{a}$ e $5^{a}$ & TOF & $2^{a}$ e $5^{a}$ & TOF \\
\hline 100 & 14,9 & 14,8 & 12,1 & 11,0 \\
\hline
\end{tabular}

Tabela 3. Painéis com fechamento lateral - AL.

\begin{tabular}{|c|c|c|c|c|}
\hline \multirow{3}{*}{$\begin{array}{l}\mathrm{F} \\
(\mathrm{kN})\end{array}$} & \multicolumn{4}{|c|}{$\Delta(\mathbf{m m})$} \\
\hline & \multicolumn{2}{|l|}{$L=6 \mathrm{~m}$} & \multicolumn{2}{|c|}{$L=7 \mathrm{~m}$} \\
\hline & $2^{a}$ e $5^{a}$ & TOF & $2^{a}$ e $5^{a}$ & TOF \\
\hline 100 & 9,4 & 9,0 & 7,8 & 7,5 \\
\hline
\end{tabular}

Tabela 4. Painéis sem fechamento lateral (PSFL) - ANL

\begin{tabular}{lllll}
\hline $\mathrm{F}$ & $\Delta \mathbf{( m m})$ & & & \\
\cline { 2 - 5 }$(\mathrm{kN})$ & $\mathbf{L}=\mathbf{4 m}$ & $\mathbf{L}=\mathbf{5 m}$ & $\mathbf{L}=\mathbf{6 m}$ & $\mathbf{L}=\mathbf{7 m}$ \\
\hline 4 & 2,5 & 2,1 & 2,0 & 1,8 \\
10 & 6,2 & 5,4 & 4,9 & 4,6 \\
40 & 25,0 & 21,6 & 20,0 & 18,5 \\
100 & 62,6 & 53,8 & 49,9 & 46,5 \\
\hline
\end{tabular}

Tabela 5. Painéis com fechamento lateral - ANL

\begin{tabular}{|c|c|c|c|c|}
\hline \multirow{3}{*}{$\begin{array}{l}\mathrm{F} \\
(\mathrm{kN})\end{array}$} & \multicolumn{4}{|c|}{$\Delta(\mathbf{m m})$} \\
\hline & \multicolumn{2}{|c|}{$L=4 m$} & \multicolumn{2}{|c|}{$\mathrm{L}=\mathbf{5 m}$} \\
\hline & $2^{a}$ e $5^{a}$ & TOF & $2^{a}$ e $5^{a}$ & TOF \\
\hline 4 & 0,6 & 0,6 & 0,5 & 0,5 \\
\hline 10 & 1,5 & 1,5 & 1,2 & 1,1 \\
\hline 40 & 6,0 & 6,0 & 4,8 & 4,4 \\
\hline 100 & 14,9 & 14,8 & 12,1 & 11,0 \\
\hline
\end{tabular}


Tabela 6. Painéis com fechamento lateral - ANL

\begin{tabular}{|c|c|c|c|c|}
\hline \multirow{3}{*}{$\begin{array}{l}\mathrm{F} \\
(\mathrm{kN})\end{array}$} & \multicolumn{4}{|c|}{$\Delta(\mathbf{m m})$} \\
\hline & \multicolumn{2}{|c|}{$L=6 m$} & \multicolumn{2}{|c|}{$L=7 m$} \\
\hline & $2^{a}$ e $5^{a}$ & TOF & $2^{a}$ e $5^{a}$ & TOF \\
\hline 4 & 0,4 & 0,3 & 0,4 & 0,3 \\
\hline 10 & 1,0 & 0,9 & 1,0 & 0,9 \\
\hline 40 & 4,0 & 3,6 & 4,0 & 3,6 \\
\hline 100 & 9,4 & 9,0 & 7,8 & 7,5 \\
\hline
\end{tabular}

Os valores dos delocamentos $(\Delta)$, obtidos inicialmente a partir das análises linear (AL) e nãolinear geométrica (ANL), efetuadas no SAP2000NonLinear, foram praticamente iguais para valores de força $(\mathrm{F})$, aplicados com valores de até $100 \mathrm{kN}$. Portanto, os valores de rigidez "K" (K1 a K4) e "G" (G1 a G8), apresentados nas tabelas $7 \mathrm{a} 9$, para os painéis analisados com e sem o fechamento lateral, considerando as distâncias longitudinais " $L$ ", foram obtidos com base nos resultados fornecidos pela análise linear (AL) dos painéis, e que estão apresentados nas tabelas $1 \mathrm{a}$ 3. Para melhor visualização do comportamento dos painéis, as curvas que relacionam a força horizontal (F) aplicada com o correspondente deslocamento $(\Delta)$, estão apresentadas nas figuras 10 a 13 , sendo que estas foram obtidas a partir dos resultados fornecidos pela analise não linear (ANL) dos painéis, conforme tabelas 4 a 6 .

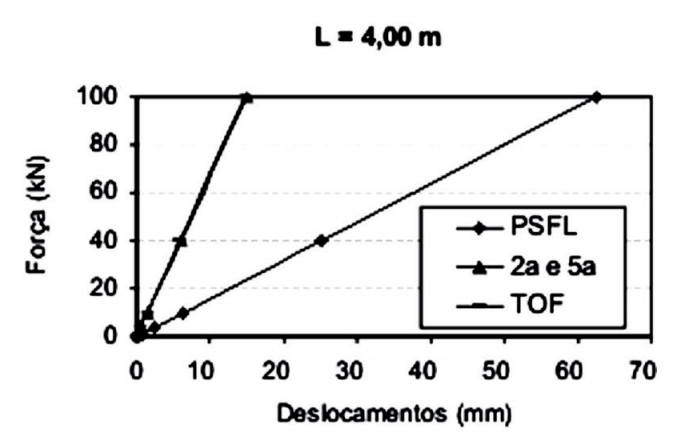

Figura 10. Curvas $\mathrm{F} \times \Delta$ para o primeiro painel analisado.

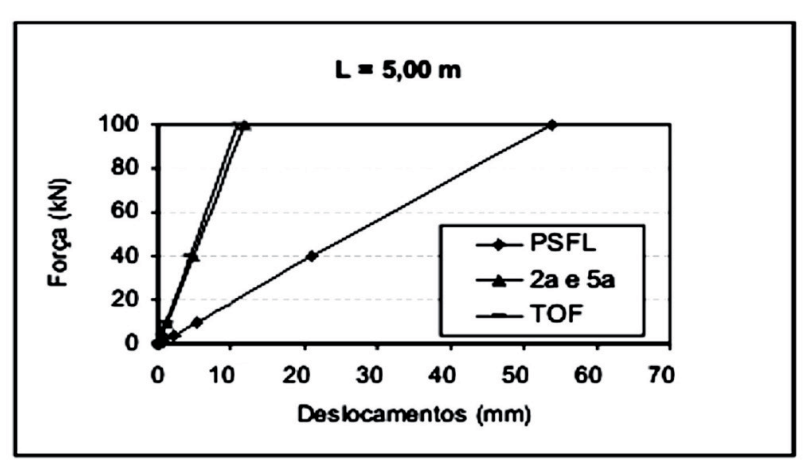

Figura 11. Curvas $\mathrm{F} \times \Delta$ para o segundo painel analisado.

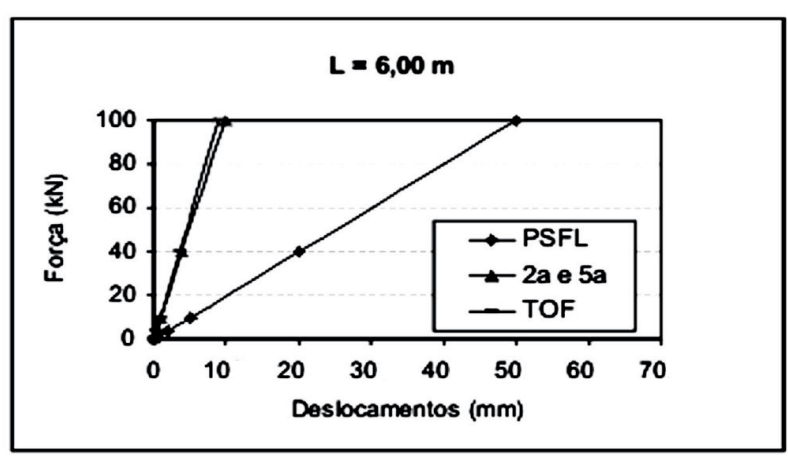

Figura 12. Curvas F x $\Delta$ para o terceiro painel analisado.

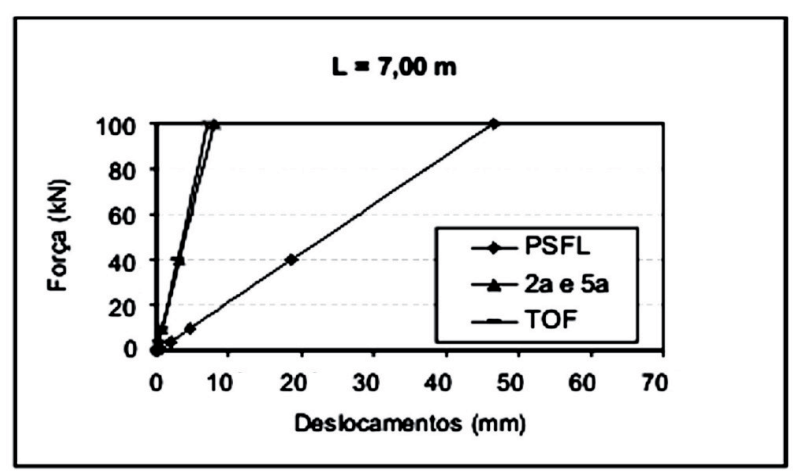

Figura 13. Curvas $\mathrm{F} x \Delta$ para o quarto painel analisado. 
Tabela 7. Rigidez $(\mathrm{K})$ dos painéis sem fechamento lateral, PSFL.

\begin{tabular}{cccc}
\hline \multicolumn{4}{c}{ Valores de $\mathbf{K}(\mathbf{k N} / \mathbf{m m})$} \\
\hline $\mathbf{L}=\mathbf{4 m}$ & $\mathbf{L}=\mathbf{5 m}$ & $\mathbf{L}=\mathbf{6 m}$ & $\mathbf{L}=\mathbf{7 m}$ \\
\hline $\mathbf{K} 1)$ & $\mathbf{( K 2 )}$ & $\mathbf{( K 3 )}$ & $\mathbf{( K 4 )}$ \\
\hline 1,6 & 1,9 & 2,0 & 2,2 \\
\hline
\end{tabular}

Tabela 8. Rigidez (G) dos painéis com o fechamento lateral.

\begin{tabular}{cccc}
\hline \multicolumn{4}{c}{ Valores de G $(\mathbf{k N} / \mathbf{m m})$} \\
\hline \multicolumn{2}{c}{$\mathbf{L}=\mathbf{4 m}$} & \multicolumn{2}{c}{$\mathbf{L}=\mathbf{5 m}$} \\
\hline $\mathbf{( G 1 )}$ & $\mathbf{( G 2 )}$ & $\mathbf{( G 3 )}$ & $\mathbf{( G 4 )}$ \\
$\mathbf{2}^{\mathrm{a}}$ e $\mathbf{5}^{\mathrm{a}}$ & TOF & $\mathbf{2}^{\mathrm{a}}$ e $\mathbf{5}^{\mathbf{a}}$ & TOF \\
\hline 6,7 & 6,8 & 8,3 & 9,1 \\
\hline
\end{tabular}

Tabela 9. Rigidez $(\mathrm{G})$ dos painéis com o fechamento lateral.

\begin{tabular}{cccc}
\hline \multicolumn{4}{c}{ Valores de G $(\mathbf{k N} / \mathbf{m m})$} \\
\hline \multicolumn{2}{c}{$\mathbf{L}=\mathbf{6 m}$} & \multicolumn{2}{c}{$\mathbf{L}=\mathbf{7 m}$} \\
\hline $\mathbf{( G 5 )}$ & $\mathbf{( G 6 )}$ & $\mathbf{( G 7 )}$ & $\mathbf{( G 8 )}$ \\
$\mathbf{2}^{\mathrm{a}}$ e 5 & TOF & $\mathbf{2}^{\mathrm{a}}$ e $\mathbf{5}^{\mathrm{a}}$ & TOF \\
\hline 10,6 & 11,1 & 12,8 & 13,3 \\
\hline
\end{tabular}

Na Figura 14 apresenta-se a comparação entre os valores dos coeficientes de rigidez " $\mathrm{K}$ " e "G", obtidos numéricamente para os painéis analisados. Os valores, neste caso, são expressos por meio de curvas que mostram o ganho de rigidez dos painéis onde foram utilizados fechamentos laterais de telhas fixas nas terças, em adição a rigidez dos paineis analisados sem o fechamento. Esses valores foram obtidos a partir das tabelas 7 a 9 .

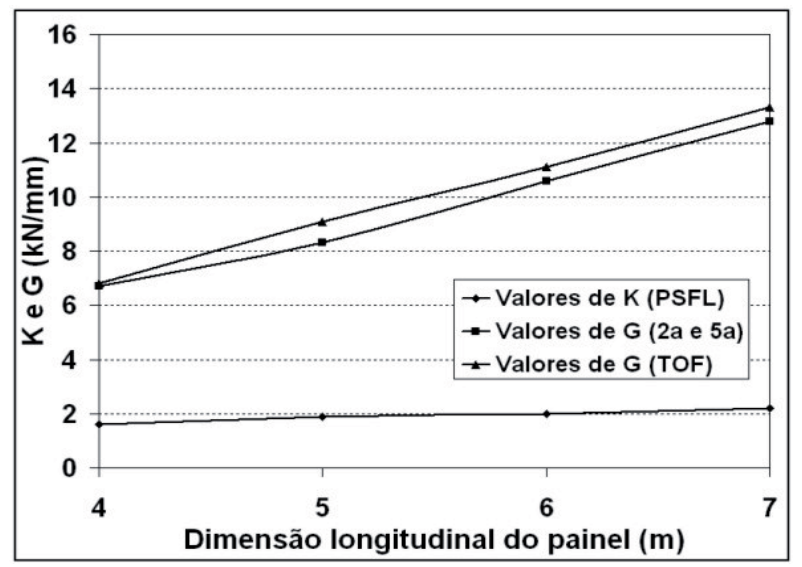

Figura 14. Comparação dos valores de rigidez $\mathrm{K}$ e G.

Tabela 10 - Forças correspondentes ao $\Delta_{\lim }=12,05 \mathrm{~mm}$.

\begin{tabular}{cccc}
\hline \multirow{2}{*}{$\mathrm{L}(\mathrm{m})$} & \multicolumn{3}{c}{$\mathbf{F}_{\lim }(\mathbf{k N})$} \\
\cline { 2 - 4 } & $\mathbf{( P S F L )}$ & $\left.\mathbf{( 2}^{\mathbf{a}} \mathbf{e} \mathbf{5}^{\mathbf{a}}\right)$ & $\mathbf{( T O F )}$ \\
\hline 4,00 & 19,30 & 80,34 & 80,33 \\
5,00 & 22,32 & 100,42 & 109,55 \\
6,00 & 24,07 & 120,52 & 133,90 \\
7,00 & 25,01 & 150,64 & 172,15 \\
\hline
\end{tabular}

Para verificação da influência das dimensões "L" na rigidez G' dos painéis com fechamento, adotou-se, com base nos valores da tabela 10, um carregamento horizontal $\mathrm{F}_{\text {ult }}$ igual a $200 \mathrm{kN}$, sendo $\mathrm{F}=0,4 . \mathrm{F}_{\text {ult }}$. O módulo de elasticidade para o aço foi de $205 \mathrm{kN} / \mathrm{mm}^{2}$ e os parâmetros utilizados na determinação da rigidez $G^{\prime}$ são apresentados nas tabelas 11 a 14. Os valores de rigidez G', obtidos a partir da equação (9), estão apresentados nas figuras 15 e 16, nas quais estes foram comparados com os valores de $\mathrm{G}$. 
Tabela 11. Momentos de inércia das armações dos painéis.

\begin{tabular}{cccc}
\hline \multicolumn{4}{c}{$\mathbf{I}\left(\mathbf{m m}^{4}\right)$} \\
\hline $\mathbf{L}=\mathbf{4 m}$ & $\mathbf{L}=\mathbf{5 m}$ & $\mathbf{L}=\mathbf{6 m}$ & $\mathbf{L}=\mathbf{7 m}$ \\
\hline $16 \times 10^{9}$ & $25 \times 10^{9}$ & $36 \times 10^{9}$ & $49 \times 10^{9}$ \\
\hline
\end{tabular}

Tabela 12. Deslocamentos numéricos horizontais $-\left(2^{\mathrm{a}}\right.$ e $\left.5^{\mathrm{a}}\right)$.

\begin{tabular}{ccccc}
\hline $\mathbf{F}$ & \multicolumn{4}{c}{$\Delta(\mathbf{m m})$} \\
\cline { 2 - 5 }$(\mathbf{k N})$ & $\mathbf{L}=\mathbf{4 m}$ & $\mathbf{L}=\mathbf{5 m}$ & $\mathbf{L}=\mathbf{6 m}$ & $\mathbf{L}=\mathbf{7 m}$ \\
\hline 80 & 11,92 & 9,68 & 7,52 & 6,24 \\
\hline
\end{tabular}

Tabela 13. Deslocamentos numéricos horizontais (TOF).

\begin{tabular}{ccccc}
\hline $\mathbf{F}$ & \multicolumn{4}{c}{$\Delta \mathbf{( m m})$} \\
\cline { 2 - 5 }$(\mathbf{k N})$ & $\mathbf{L}=\mathbf{4 m}$ & $\mathbf{L}=\mathbf{5 m}$ & $\mathbf{L}=\mathbf{6 m}$ & $\mathbf{L}=\mathbf{7 m}$ \\
\hline 80 & 11,84 & 8,80 & 7,20 & 6,00 \\
\hline
\end{tabular}

Tabela 14. Deslocamentos provocados por flexão.

\begin{tabular}{ccccc}
\hline $\mathbf{F}$ & \multicolumn{4}{c}{$\boldsymbol{\Delta}_{\mathbf{f}}(\mathbf{m m})$} \\
\cline { 2 - 5 }$(\mathbf{k N})$ & $\mathbf{L}=\mathbf{4 m}$ & $\mathbf{L}=\mathbf{5} \mathbf{m}$ & $\mathbf{L}=\mathbf{6} \mathbf{m}$ & $\mathbf{L}=\mathbf{7 m}$ \\
\hline 80 & $9,6 \times 10^{-1}$ & $6,1 \times 10^{-1}$ & $4,2 \times 10^{-1}$ & $3,1 \times 10^{-1}$ \\
\hline
\end{tabular}

Tabela 15. Deslocamentos por cisalhamento $-\left(2^{\mathrm{a}}\right.$ e $\left.5^{\mathrm{a}}\right)$.

\begin{tabular}{ccccc}
\hline $\mathbf{F}$ & \multicolumn{4}{c}{$\boldsymbol{\Delta}_{\mathbf{s}}(\mathbf{m m})$} \\
\cline { 2 - 5 }$(\mathbf{k N})$ & $\mathbf{L}=\mathbf{4 m}$ & $\mathbf{L}=\mathbf{5 m}$ & $\mathbf{L}=\mathbf{6 m}$ & $\mathbf{L}=\mathbf{7 m}$ \\
\hline 80 & 10,96 & 9,07 & 7,09 & 5,93 \\
\hline
\end{tabular}

Tabela 16. Deslocamentos por cisalhamento - (TOF).

\begin{tabular}{ccccc}
\hline $\mathbf{F}$ & \multicolumn{4}{c}{$\boldsymbol{\Delta}_{\mathbf{s}}(\mathbf{m m})$} \\
\cline { 2 - 5 }$(\mathbf{k N})$ & $\mathbf{L}=\mathbf{4 m}$ & $\mathbf{L}=\mathbf{5 m}$ & $\mathbf{L}=\mathbf{6 m}$ & $\mathbf{L}=\mathbf{7 m}$ \\
\hline 80 & 10,88 & 8,19 & 6,77 & 5,69 \\
\hline
\end{tabular}

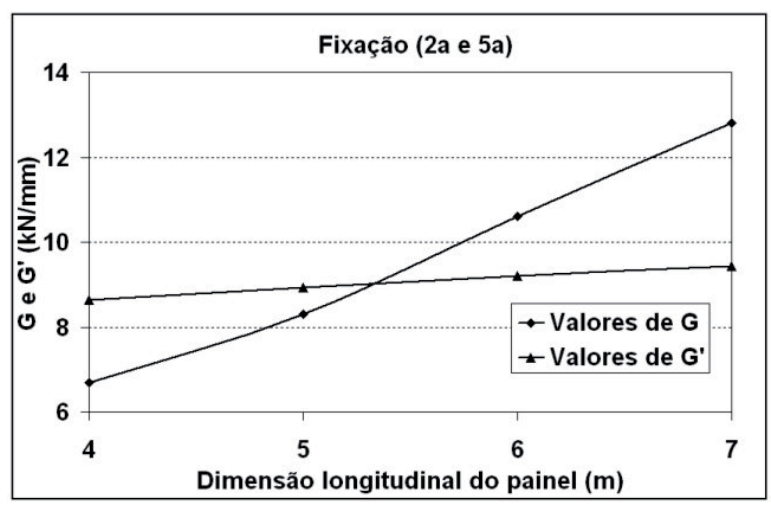

Figura 15. Comparação dos valores de rigidez G e G’.

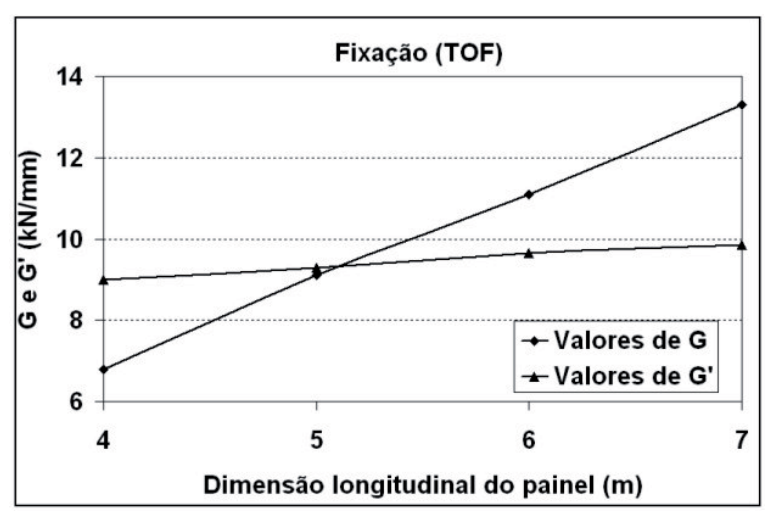

Figura 16. Comparação dos valores de rigidez $\mathrm{G}$ e G'.

As tabelas 17 e 18 apresentam as relações entre as parcelas de rigidez dos painéis contraventados somente com tirantes com relação às parcelas de rigidez totais dos painéis com fechamentos.

Tabela 17. Relações entre as parcelas de rigidez dos painéis.

\begin{tabular}{cccc}
\hline \multicolumn{5}{c}{ K/G (\%) } \\
\hline \multicolumn{3}{c}{$\mathbf{L}=\mathbf{4 m}$} & \multicolumn{2}{c}{$\mathbf{L}=\mathbf{5 m}$} \\
\hline $\mathbf{K 1} / \mathbf{G 1}$ & $\mathbf{K 1} / \mathbf{G} \mathbf{2}$ & $\mathbf{K 2} / \mathbf{G 3}$ & $\mathbf{K 2} / \mathbf{G 4}$ \\
\hline 0,24 & 0,24 & 0,21 & 0,21 \\
\hline
\end{tabular}


Tabela 18. Relações entre as parcelas de rigidez dos painéis.

\begin{tabular}{cccc}
\hline \multicolumn{5}{c}{ K/G (\%) } \\
\hline \multicolumn{3}{c}{ L=6m } & \multicolumn{2}{c}{$\mathbf{L}=\mathbf{7 m}$} \\
\hline K3/G5 & K3/G6 & K4/G7 & K4/G8 \\
\hline 0,19 & 0,18 & 0,17 & 0,16 \\
\hline
\end{tabular}

Observa-se que a contribuição da rigidez telha-terça na contenção longitudinal dos painéis correspondeu em média a $81 \%$.

\section{Discussão}

As relações entre as forças horizontais (F) aplicadas e os correspondentes deslocamentos $(\Delta)$ obtidos apresentaram relações praticamente lineares crescentes para os níveis de força aplicados na analise não linear geométrica.

Quando comparados os valores de rigidez $\mathrm{K}$ e $\mathrm{G}$, observou-se o ganho de rigidez proporcionado pela fixação telha-terça dos painéis analisados com fechamento. Além disso, os dois tipos de fixação das telhas analisados não apresentaram grandes diferenças em termos de rigidez $\mathrm{G}$.

Quando comparados os valores de rigidez $\mathrm{G}$ e G' observou-se que os valores de $G$ foram menores que os valores de G' para os paineis de $4 \mathrm{~m}$. Os valores de rigidez $G$ ' e $G$ tenderam a se igualar para os painéis de $5 \mathrm{~m}$, sendo que para os painéis de $6 \mathrm{~m}$ e $7 \mathrm{~m}$ os valores de $\mathrm{G}$ mostraram-se maiores que os de G'. Esse comportamento deve-se ao fato de que na determinação dos valores de $\mathrm{G}$, o deslocamento horizontal $(\Delta)$ total considera os deslocamentos por flexão $\left(\Delta_{\mathrm{f}}\right)$ como também por cisalhamento $\left(\Delta_{\mathrm{s}}\right)$. Na determinação de G' são considerados apenas os deslocamentos devidos ao cisalhamento $\left(\Delta_{\mathrm{s}}\right) \mathrm{e}$, neste caso, com o aumento da dimensão " $L$ " a relação $h / L$ faz com que haja a diminuição do valor de G'.

\section{Conclusões}

Autilização do fechamento lateral, a partir dafixação das telhas nas terças, contribuiu significativamente para a contenção longitudinal dos painéis, em virtude do aumento do coeficiente de rigidez dos mesmos. A ação diafragma proporcionada pelo conjunto telhaterça aumentou a rigidez dos painéis analisados com fechamento em até $84 \%$.

Os acréscimos de rigidez proporcionados pela fixação telha-terça em painéis com 4,0, 5,0, 6,0 e 7,0 metros, com fixação do tipo $\left(2^{\mathrm{a}}\right.$ e $\left.5^{\mathrm{a}}\right)$ foram, respectivamente, $76 \%, 79 \%, 81 \%$ e $83 \%$. Para os painéis com fixação do tipo (TOF), os acréscimos de rigidez foram de $76 \%, 79 \%, 82 \%$ e $84 \%$.

$\mathrm{Na}$ prática, as telhas fixadas nas $2^{\mathrm{a}}$ e $5^{\mathrm{a}}$ ondas baixas das terças se justificam em função da economia de material (parafusos) que pode ser obtida com a diminuição do número de pontos de fixação. A rigidez proporcionada pelos dois tipos de fixação, $\left(2^{\mathrm{a}}\right.$ e $5^{\mathrm{a}}$ ) e (TOF), analisados não apresentaram diferenças significativas.

Os resultados de rigidez aqui apresentados foram obtidos a partir de análise numérica, necessitando de comprovação experimental.

\section{Agradecimentos}

À FAPESP pelo apoio financeiro da pesquisa.

\section{Referências}

ASSOCIAÇÃO BRASILEIRA DE NORMAS TÉCNICAS. NBR 14762: Dimensionamento de estruturas de aço constituídas por perfis formados a frio Procedimento. Rio de Janeiro: ABNT, 2001. 77 p.

BARALDI, L. T. Ação diafragma de cobertura aplicada as construções em pórticos de madeira. 2001. $77 \mathrm{f}$. Tese (Doutorado em Engenharia de Estruturas) - Escola de Engenharia de São Carlos, Universidade de São Paulo, São Carlos, 2001. 
BASAGLIA, C. D. Sobre o comportamento estrutural de telhas de aço considerando a interação com as telhas. 2004. Dissertação (Mestrado em Engenharia de Estruturas) - Escola de Engenharia de São Carlos, Universidade de São Paulo, São Carlos.

BELLEI, I. H. Edifícios industriais em aço: Projeto e Cálculo. São Paulo: Pini, 1994.

COMPUTERS AND STRUCTURES, INC. - "SAP 2000NonLinear Version 7.42: Structural Analysis Program", Berkeley, California, 1997.

ENGLEKIRK, R. Steel Structures-Controlling Behavior Through Design. New York: John Wiley \& Sons, 1994. $807 \mathrm{p}$.
METFORM. Perfis de aço formados a frio. Betin-MG: Metform, 2002a. Catálogo. Disponível em http://www. metform.com.br/website/produtos/serie_tipos.php. Acesso em 26 de junho de 2009.

. Telhas de aço. Betin-MG: Metform, 2002b.

Catálogo. Disponível em http://www.metform.com.br/ website/produtos/telhas_dadostecnicos.php. Acesso em 26 de junho de 2009.

YU, W. Cold-formed Steel Design. New York: WileyInterscience, $1985.545 \mathrm{p}$. 